\title{
Thermodynamics and heavy-quark free energies at finite temperature and density with two flavors of improved Wilson quarks
}

\author{
Y. Maezawa*, T. Hatsuda \\ Department of Physics, The University of Tokyo, \\ Bunkyo-ku, Tokyo 113-0033, Japan \\ E-mail: maezawa@nt.phys.s.u-tokyo.ac.jp
}

\section{S. Aoki, K. Kanaya, T. Umeda}

Graduate School of Pure and Applied Sciences, University of Tsukuba, Tsukuba, Ibaraki 305-8571, Japan

\section{S. Ejiri}

Physics Department, Brookhaven National Laboratory,

Upton, New York 11973, USA

\section{N. Ishii and N. Ukita}

Center for Computational Sciences, University of Tsukuba,

Tsukuba, Ibaraki 305-8577, Japan

\begin{abstract}
Thermodynamics of two-flavor QCD at finite temperature and density is studied on a $16^{3} \times 4$ lattice, using a renormalization group improved gauge action and the clover improved Wilson quark action. In the simulations along lines of constant $m_{\mathrm{PS}} / m_{\mathrm{V}}$, we calculate the Taylor expansion coefficients of the heavy-quark free energy with respect to the quark chemical potential $\left(\mu_{q}\right)$ up to the second order. By comparing the expansion coefficients of the free energies between quark $(Q)$ and antiquark $(\bar{Q})$, and between $Q$ and $Q$, we find a characteristic difference at finite $\mu_{q}$ due to the first order coefficient of the Taylor expansion. We also calculate the quark number and isospin susceptibilities, and find that the second order coefficient of the quark number susceptibility shows enhancement around the pseudo-critical temperature.
\end{abstract}

The XXV International Symposium on Lattice Field Theory

July 30 - August 42007

Regensburg, Germany

\footnotetext{
* Speaker.
} 


\section{Introduction}

We study QCD thermodynamics at finite temperature $(T)$ and quark chemical potential $\left(\mu_{q}\right)$ with two-flavors of dynamical quarks. Then, we calculate the Taylor expansion coefficients of physical quantities in the simulations at $\mu_{q}=0$, and investigate thermodynamic properties at small $\mu_{q}$ region. In this report, we present current status of two topics: heavy-quark free energy and fluctuation at finite $\mu_{q}$. The former is related to the inter-quark interaction in quark-gluon plasma, and the latter is related to the existence of the critical point in $\left(T, \mu_{q}\right)$ plane.

\section{Lattice action and simulation parameters}

We employ a renormalization group improved gauge action and a clover improved Wilson quark action with two flavors. The numerical simulation is performed on a lattice with a size of $N_{s}^{3} \times N_{t}=16^{3} \times 4$ along lines of constant physics, i.e. lines of constant $m_{\mathrm{PS}} / m_{\mathrm{V}}$ (the ratio of pseudoscalar and vector meson masses) at $T=0$ in the space of simulation parameters. Two values of $m_{\mathrm{PS}} / m_{\mathrm{V}}$ are taken: 0.65 and 0.80 with the temperature range of $T / T_{p c} \sim 0.82-4.0$ and $0.76-3.0$, respectively, where $T_{p c}$ is the pseudo-critical temperature along the line of constant physics. The number of trajectories for each run after thermalization is 5000-6000, and we measure physical quantities at every 10 trajectories. Details of the lines of constant physics with the same actions are summarized in Ref. [1, 2]. To calculate derivatives of the quark determinant with respect to $\mu_{q}$, we use the random noise method introduced in Ref. [3] with the number of noise of 100-200.

\section{Heavy quark free energy and Debye screening mass}

The heavy-quark free energy in the QCD medium is one of the important quantities to characterize the properties of the quark-gluon plasma. Especially, the properties of the free energies at finite $T$ and $\mu_{q}$ may be related to the fate of the charmoniums and bottomoniums in relativistic heavy ion collisions. Precise studies of the heavy-quark free energy in two-flavors QCD have been done with the improved Wilson quark action at $\mu_{q}=0$ [2]. Properties of the free energy between static-quark $(Q)$ and -antiquark $(\bar{Q})$ at finite $\mu_{q}$ have been previously investigated by using the Taylor expansion method using an improved staggered quark action [4]. In this section, we show the Taylor expansion coefficients of the free energy between not only $Q$ and $\bar{Q}$, but also $Q$ and $Q$, up to 2nd-order of $\mu_{q}$.

The free energy of static quarks on the lattice is described by the correlations of the Polyakov loop: $\Omega(\mathbf{x})=\prod_{\tau=1}^{N_{t}} U_{4}(\tau, \mathbf{x})$ where the $U_{\mu}(\tau, \mathbf{x}) \in \mathrm{SU}(3)$ is the link variable. With an appropriate gauge fixing (e.g. the Coulomb gauge fixing), one can define the free energy in various color channels separately: the color singlet $Q \bar{Q}$ channel (1), the color octet $Q \bar{Q}$ channel (8), the color anti-triplet $Q Q$ channel $\left(\mathbf{3}^{*}\right)$, and the color sextet $Q Q$ channel (6), given as follows.

$$
\begin{aligned}
e^{-F^{1}(r, T) / T} & =\frac{1}{3}\left\langle\operatorname{Tr} \Omega^{\dagger}(\mathbf{x}) \Omega(\mathbf{y})\right\rangle, \\
e^{-F^{\mathbf{8}}(r, T) / T} & =\frac{1}{8}\left\langle\operatorname{Tr} \Omega^{\dagger}(\mathbf{x}) \operatorname{Tr} \Omega(\mathbf{y})\right\rangle-\frac{1}{24}\left\langle\operatorname{Tr} \Omega^{\dagger}(\mathbf{x}) \Omega(\mathbf{y})\right\rangle, \\
e^{-F^{\mathbf{6}}(r, T) / T} & =\frac{1}{12}\langle\operatorname{Tr} \Omega(\mathbf{x}) \operatorname{Tr} \Omega(\mathbf{y})\rangle+\frac{1}{12}\langle\operatorname{Tr} \Omega(\mathbf{x}) \Omega(\mathbf{y})\rangle,
\end{aligned}
$$



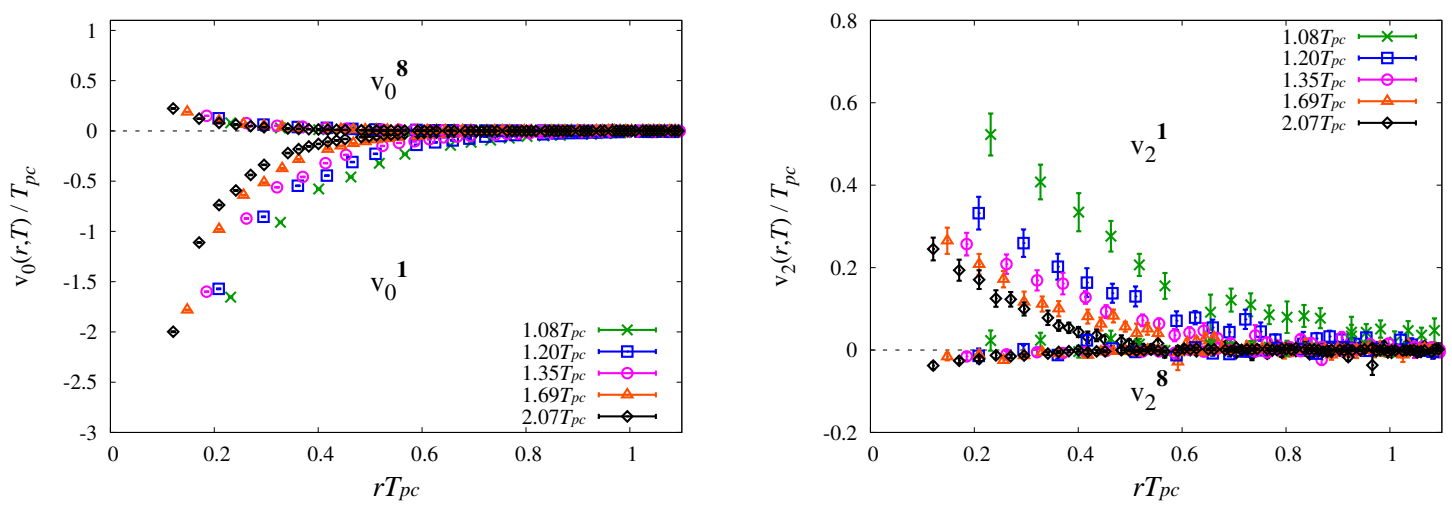

Figure 1: Results of $v_{0}^{M}$ (left) and $v_{2}^{M}$ (right) for $Q \bar{Q}$ channel above $T_{p c}$ at $m_{\mathrm{PS}} / m_{\mathrm{V}}=0.80$.

$$
e^{-F^{3^{*}}(r, T) / T}=\frac{1}{6}\langle\operatorname{Tr} \Omega(\mathbf{x}) \operatorname{Tr} \Omega(\mathbf{y})\rangle-\frac{1}{6}\langle\operatorname{Tr} \Omega(\mathbf{x}) \Omega(\mathbf{y})\rangle,
$$

where $r=|\mathbf{x}-\mathbf{y}|$.

For $T>T_{p c}$, we introduce normalized free energies $\left(V^{\mathbf{1}}, V^{\mathbf{8}}, V^{\mathbf{6}}, V^{\mathbf{3}^{*}}\right)$ such that they vanish at large distances. This is equivalent to define the free energies by dividing the right-hand side of Eq. (3.1)-(3.4) by $\langle\operatorname{Tr} \Omega\rangle\left\langle\operatorname{Tr} \Omega^{\dagger}\right\rangle$ for $Q \bar{Q}$ free energies and $\langle\operatorname{Tr} \Omega\rangle^{2}$ for $Q Q$ free energies.

The Taylor expansion of normalized free energies with respect to $\mu_{q} / T$ is described as a power series up to 2 nd order:

$$
V^{M}\left(r, T, \mu_{q}\right)=v_{0}^{M}+v_{1}^{M}\left(\frac{\mu_{q}}{T}\right)+v_{2}^{M}\left(\frac{\mu_{q}}{T}\right)^{2}+O\left(\mu_{q}^{3}\right),
$$

where $M$ is the color channel. The coefficients, $v_{n}^{M}$, can be evaluated by expanding the quark determinant of partition function in powers of $\mu_{q}$, then the normalization of the free energies by $\langle\operatorname{Tr} \Omega\rangle$ is also taken order by order of $\mu_{q}$.

One should note that the color singlet and octet channels do not have the odd orders in the Taylor expansion since the free energies for both channels are symmetric with respect to $\mu_{q}$. In other words, the free energies between $Q$ and $\bar{Q}$ are invariant under the charge conjugation. On the other hand, the color sextet and antitriplet channels has the odd orders since the $Q Q$ free energies are not invariant under the charge conjugation.

The expansion coefficients of the normalized free energies for the color singlet and octet $Q \bar{Q}$ channels are shown in Fig. 1 for $v_{0}^{M}$ (left) and $v_{2}^{M}$ (right) at $m_{\mathrm{PS}} / m_{\mathrm{V}}=0.80$ for several temperatures. Those for the color sextet and antitriplet $Q Q$ channels are shown in Fig. 2 for $v_{0}^{M}$ (left) and $v_{1}^{M}$ (right) and in Fig. 3 for $v_{2}^{M}$. It have been found in Ref. [2] that the inter-quark interaction is "attractive" in the color singlet and antitriplet channels and is "repulsive" in the color octet and sextet channels at $\mu_{q}=0$. We find that, both in high and low temperatures, the sign of $v_{1}^{M}$ is the same with that of $v_{0}^{M}$, whereas the sign of a $v_{2}^{M}$ is the opposite of that of $v_{0}^{M}$, i.e. $v_{1}^{M} \cdot v_{0}^{M}>0$ (only for $Q Q$ free energies) and $v_{2}^{M} \cdot v_{0}^{M}<0$. This means that the inter-quark interaction between $Q$ and $\bar{Q}$ becomes weak, whereas that between $Q$ and $Q$ becomes strong in the leading-order of $\mu_{q}$. In other words, $Q \bar{Q}(Q Q)$ free energies are screened (anti-screened) by contributions of the internal quarks induced by finite $\mu_{q}$. 

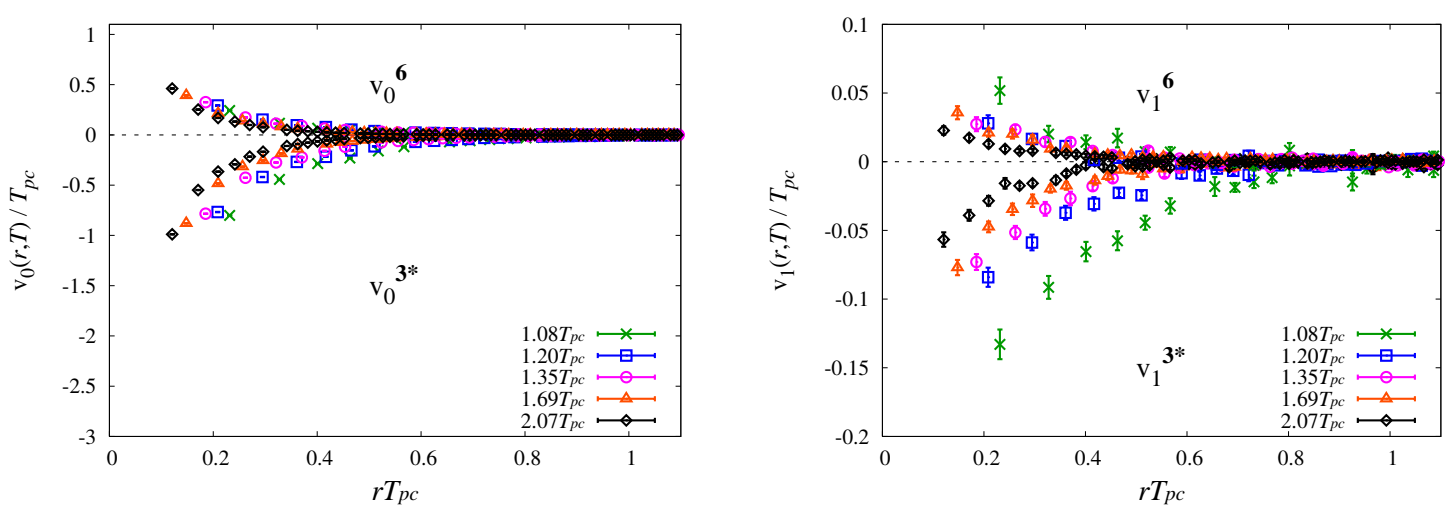

Figure 2: Results of $v_{0}^{M}$ (left) and $v_{1}^{M}$ (right) for $Q Q$ channel above $T_{p c}$ at $m_{\mathrm{PS}} / m_{\mathrm{V}}=0.80$.

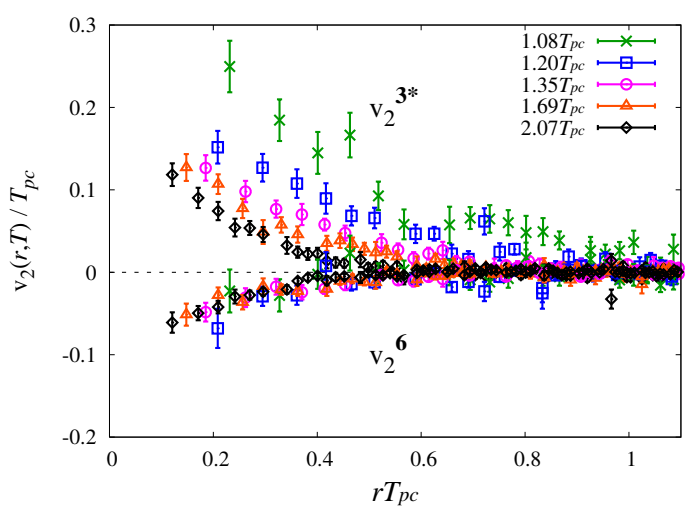

Figure 3: Results of $v_{2}^{M}$ for $Q Q$ channel above $T_{p c}$ at $m_{\mathrm{PS}} / m_{\mathrm{V}}=0.80$

In order to study the screening effect in each color channel, we fit the normalized free energies by a screened Coulomb form,

$$
V^{M}\left(r, T, \mu_{q}\right)=C(M) \frac{\alpha_{\mathrm{eff}}\left(T, \mu_{q}\right)}{r} e^{-m_{D}\left(T, \mu_{q}\right) r},
$$

where $\alpha_{\text {eff }}\left(T, \mu_{q}\right)$ and $m_{D}\left(T, \mu_{q}\right)$ are the effective running coupling and the Debye screening mass, respectively. We assume that contributions of finite $\mu_{q}$ appear only in $\alpha_{\text {eff }}$ and $m_{D}$. The Casimir factor $C(M) \equiv\left\langle\sum_{a=1}^{8} t_{1}^{a} \cdot t_{2}^{a}\right\rangle_{M}$ for color channel $M$ is given by

$$
C(\mathbf{1})=-\frac{4}{3}, \quad C(\mathbf{8})=\frac{1}{6}, \quad C(\mathbf{6})=\frac{1}{3}, \quad C\left(\mathbf{3}^{*}\right)=-\frac{2}{3} .
$$

We assume that $m_{D}\left(T, \mu_{q}\right)$ is also expressed as a power series of $\mu_{q} / T$ :

$$
m_{D}=m_{D, 0}+m_{D, 2}\left(\frac{\mu_{q}}{T}\right)^{2}+O\left(\mu_{q}^{4}\right),
$$

where we use the fact that the Debye screening mass does not have the odd powers in the Taylor expansion because it is related to the self-energy of the two-point correlation of the gauge fields which is symmetric when $\mu_{q} \rightarrow-\mu_{q}$. Relations of coefficients between $V^{M}$ and $m_{D}$ are given by comparing each order of $\mu_{q}$. At $\mu_{q}=0$, the relation is the same as that adopted in Ref. [2]:

$$
v_{0}(r, T)=C(M) \frac{\alpha_{\mathrm{eff}, 0}(T)}{r} e^{-m_{D, 0}(T) r} .
$$



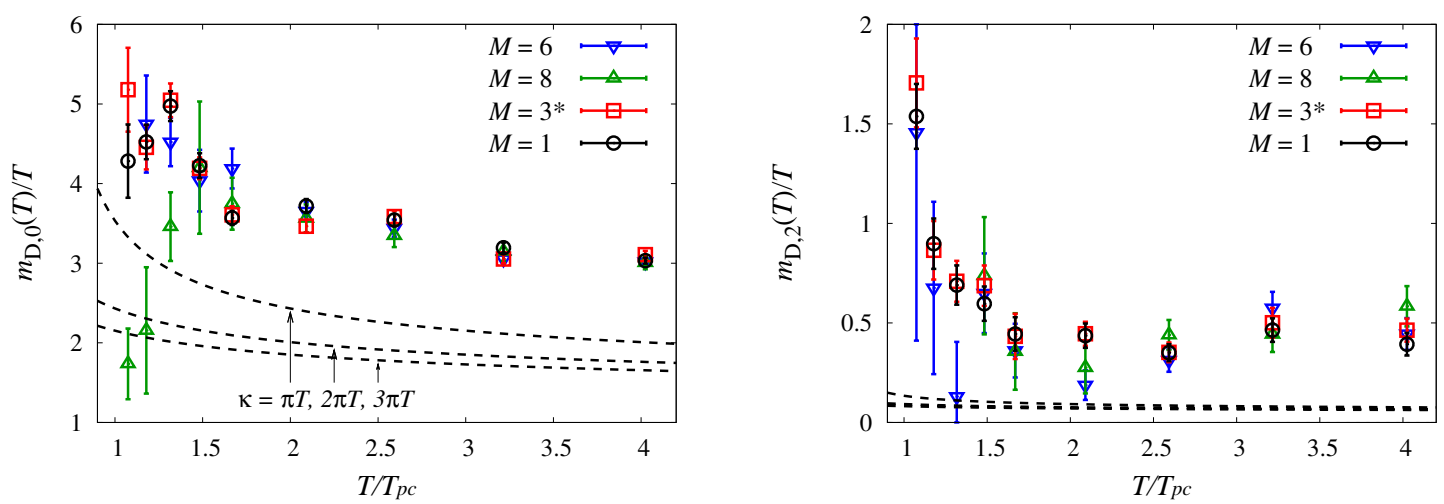

Figure 4: Results of $m_{D, 0}$ (left) and $m_{D, 2}$ for each color channel at $m_{\mathrm{PS}} / m_{\mathrm{V}}=0.65$. Dashed lines are prediction from a leading-order of the thermal perturbation theory with the renormalization points of $\kappa=\pi T$, $2 \pi T$ and $3 \pi T$.

For the second order coefficients, we obtain,

$$
\frac{v_{2}}{v_{0}} \simeq-m_{D, 2} r
$$

At large distances, we estimate the coefficients of Debye mass by fitting the normalized free energies for each color channel with the formulae (3.9) and (3.10).

Figure 4 shows the results of the $m_{D, 0}(T)$ (left) and $m_{D, 2}(T)$ (right) for $m_{\mathrm{PS}} / m_{\mathrm{V}}=0.65$. Similar behavior in both results are obtained for $m_{\mathrm{PS}} / m_{\mathrm{V}}=0.80$. We find that there is no significant channel dependence in both coefficients at sufficiently high temperatures $\left(T \gtrsim 2 T_{p c}\right)$. In other words, the channel dependence of the free energy at high temperature can be well absorbed in the Casimir factor.

Let us compare the Debye screening mass at finite $\mu_{q}$ on the lattice with that predicted in the thermal perturbation theory. The 2-loop running coupling is given by

$$
g_{21}^{-2}(\kappa)=\beta_{0} \ln \left(\frac{\kappa}{\Lambda}\right)^{2}+\frac{\beta_{1}}{\beta_{0}} \ln \ln \left(\frac{\kappa}{\Lambda}\right)^{2}
$$

where $\kappa$ is the renormalization point. The argument in the logarithms can be written as $\kappa / \Lambda=$ $(\kappa / T)\left(T / T_{p c}\right)\left(T_{p c} / \Lambda\right)$ with $\Lambda=\Lambda_{\overline{M S}}^{N_{f}=2} \simeq 261 \mathrm{MeV}$ [5] and $T_{p c} \simeq 171 \mathrm{MeV}$ [1]. We assume $\kappa$ to be in a range $\pi T$ to $3 \pi T$. Therefore, $g_{21}$ can be viewed as a function of $T / T_{p c}$. In the leading order of the thermal perturbation theory, the Debye screening mass with $g_{21}$ is given by

$$
m_{D}^{\mathrm{LO}}\left(T, \mu_{q}\right)=g_{21}(T)\left\{\left(1+\frac{N_{f}}{6}\right) T^{2}+\frac{N_{f}}{2 \pi^{2}} \mu_{q}^{2}\right\}^{1 / 2} .
$$

Therefore, the leading-order expansion coefficients in the thermal perturbation theory are given by

$$
m_{D, 0}^{\mathrm{LO}}(T)=\sqrt{1+\frac{N_{f}}{6}} g_{21}(T) T, \quad m_{D, 2}^{\mathrm{LO}}(T)=\frac{1}{4 \pi^{2}} \frac{N_{f}}{\sqrt{1+N_{f} / 6}} g_{21}(T) T .
$$

The dashed lines in Fig. 4 are the results of $m_{D, 0}^{\mathrm{LO}}$ and $m_{D, 2}^{\mathrm{LO}}$ for $\kappa=\pi T, 2 \pi T$ and $3 \pi T$. We find that the lattice results are larger than the leading-order thermal perturbation for both coefficients 
of the Debye mass. Since it is known that the next-to-leading-order in the thermal perturbation with respect to $T$ compensates for such discrepancy in the case at $\mu_{q}=0$ [2], the higher order contributions in the perturbation theory with respect to $\mu_{q}$ could help us to understand our results on the lattice.

\section{Hadronic fluctuations at finite $\mu_{q}$}

Hadronic fluctuations at finite density are observables closely related to the critical point in the $\left(T, \mu_{q}\right)$ plane and may be experimentally detected by an event-by-event analysis of heavy ion collisions. The fluctuations can also be studied by numerical simulations of lattice QCD calculating the quark number and isospin susceptibilities, $\chi_{q}$ and $\chi_{I}$. They correspond to the second derivatives of the pressure with respect to $\mu_{q}$ and $\mu_{I}$, where $\mu_{I}$ is the isospin chemical potential. From a phenomenological argument in the sigma model, $\chi_{q}$ is singular at the critical point, whereas $\chi_{I}$ shows no singularity there [6].

In this section, we calculate the $\chi_{q}$ and $\chi_{I}$ and their second derivatives with respect to $\mu_{q}$ and $\mu_{I}$ at $\mu_{q}=\mu_{I}=0$. (Note that the odd derivatives are zero at $\mu_{q}=0$.) The details of the calculations are reported in Ref. [3]. The left panel of Fig. 5 shows $\chi_{q} / T^{2}$ (circle) and $\chi_{I} / T^{2}$ (square) at $m_{\mathrm{PS}} / m_{\mathrm{V}}=$ 0.8 and $\mu_{q}=\mu_{I}=0$ as functions of $T / T_{p c}$. We find that $\chi_{q} / T^{2}$ and $\chi_{I} / T^{2}$ increase sharply at $T_{p c}$, in accordance with the expectation that the fluctuations in the QGP phase are much larger than those in the hadron phase. Their second derivatives $\partial^{2}\left(\chi_{q} / T^{2}\right) / \partial\left(\mu_{q} / T\right)^{2}$ and $\partial^{2}\left(\chi_{I} / T^{2}\right) / \partial\left(\mu_{q} / T\right)^{2}$ are shown in Fig. 5 (right). We find that basic features are quite similar to those found previously with the p4-improved staggered fermions [7]. $\partial^{2}\left(\chi_{I} / T^{2}\right) / \partial\left(\mu_{q} / T\right)^{2}$ remains small around $T_{p c}$, suggesting that there are no singularities in $\chi_{I}$ at non-zero density. On the other hand, we expect a large enhancement in the quark number fluctuations near $T_{p c}$ as approaching the critical point in the $\left(T, \mu_{q}\right)$ plane. The dashed line in Fig. 5 (right) is a prediction from the hadron resonance gas model, $\partial^{2} \chi_{q} / \partial \mu_{q}^{2} \approx 9 \chi_{q} / T^{2}$. Although current statistical errors in Fig. 5 (right) are still large, we find that $\partial^{2}\left(\chi_{q} / T^{2}\right) / \partial\left(\mu_{q} / T\right)^{2}$ near $T_{p c}$ is much larger than that at high temperature. At the right end of the figure, values of free quark-gluon gas (Stefan-Boltzmann gas) for $N_{t}=4$ and for $N_{t}=\infty$ limit are shown. Since the lattice discretization error in the equation of state is known to be large at $N_{t}=4$ with our quark action, we need to extend our study to larger $N_{t}$ for the continuum extrapolation.

\section{Summary}

We presented current status of thermodynamics of two-flavor QCD with the renormalization group improved gauge action and the clover improved Wilson quark action. Simulations were performed on a $16^{3} \times 4$ lattice and along lines of constant $m_{\mathrm{PS}} / m_{\mathrm{V}}=0.65$ and 0.80 .

The properties of the heavy-quark free energies at finite $\mu_{q}$ were studied in the Taylor expansion method up to 2 nd order of $\mu_{q}$. We find that there is a characteristic difference between $Q \bar{Q}$ and $Q Q$ free energies due to the first order coefficient of the Taylor expansion. It suggests that the interquark interaction between $Q$ and $\bar{Q}\left(Q\right.$ and $Q$ ) become week (strong) in the leading-order of $\mu_{q}$. We also extract the expansion coefficients of the Debye screening mass for each color channel up to 2nd order of $\mu_{q}$. The Debye mass shows no significant channel dependence at $T \gtrsim 2 T_{p c}$, whereas, 

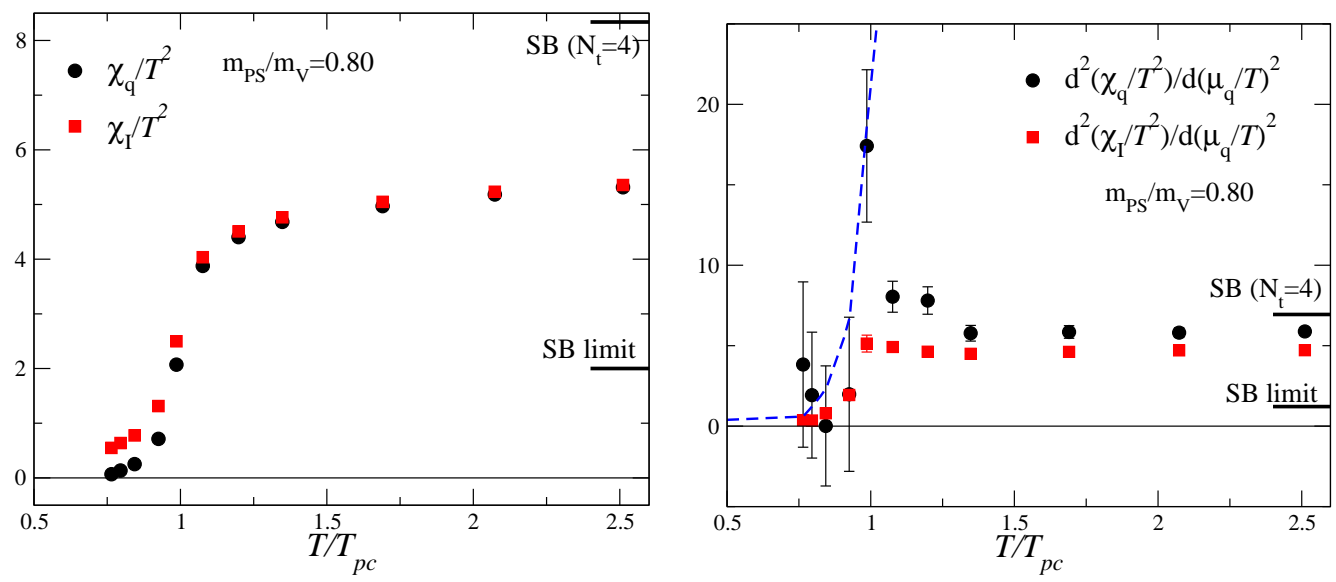

Figure 5: Left: Quark number (circle) and isospin (square) susceptibilities at $\mu_{q}=\mu_{I}=0$. Right: The second derivatives of these susceptibilities.

we find disagreement with leading-order predictions of the thermal perturbation theory. Since it is known that the next-to-leading-order of the thermal perturbation with respect to $T$ well reproduces the Debye mass on a lattice at $\mu_{q}=0$ [2], the calculations of the higher order perturbation with respect to $\mu_{q}$ will help us to understand the results on the lattice.

The fluctuations of quark number and isospin densities were also discussed. Although the statistical errors are still large, we find that $\chi_{q}$ seems to increase rapidly near $T_{p c}$ as $\mu_{q}$ increases, whereas the increase of $\chi_{I}$ is not large near $T_{p c}$. These behaviors qualitatively agree with the previous results obtained with the p4-improved staggered fermions.

\section{Acknowledgments}

This work is in part supported by Grants-in-Aid of the Japanese MEXT (Nos. 13135204, $15540251,17340066,18540253)$. YM is supported by JSPS, and SE is supported by the U.S. Department of Energy (DE-AC02-98CH1-886). This work is in part supported also by ACCC, Univ. of Tsukuba, and the Large Scale Simulation Program No.06-19 (FY2006) of KEK.

\section{References}

[1] A. Ali Khan et al. (CP-PACS Collaboration), Phys. Rev. D63 (2001) 034502; 64 (2001) 074510.

[2] Y. Maezawa et al. (WHOT-QCD Collaboration), Phys. Rev. D75, (2007) 074501.

[3] S. Ejiri et al. in proceedings of Lattice 2006. POS (LAT2006) 132

[4] M. Döring, S. Ejiri, O. Kaczmarek, F. Karsch and E. Laermann, Eur. Phys. J. C46, 179 (2006).

[5] M. Gockeler et al., Phys. Rev. D73 (2006) 014513.

[6] Y. Hatta and M. A. Stephanov, Phys. Rev. Lett.91 (2003) 102003.

[7] C.R. Allton et al., Phys. Rev. D68 (2003) 014507; 71 (2005) 054508. 